\title{
25 Research Soure \\ Identification of an Autophagy-Related IncRNA Prognostic Signature and Related Tumor Immunity Research in Lung Adenocarcinoma
}

\section{Hang Chen}

Ningbo University Medical School

\section{Menglu Sang}

Ningbo Medical Treatment Centre Li Huili Hospital

\section{Saiqi Ni}

Ningbo City First Hospital

Yao Lin

Ningbo University Medical School

\section{Chengfang Wu}

Ningbo University Medical School

\section{Yinyu Mu}

Ningbo Medical Treatment Centre Li Huili Hospital

Kaitai Liu

Ningbo Medical Treatment Centre Li Huili Hospital

\section{Shibo Wu}

Ningbo Medical Treatment Centre Li Huili Hospital

Ni Li

Ningbo Medical Treatment Centre Li Huili Hospital

Guodong Xu ( $\nabla$ xuguodong@nbu.edu.cn)

Ningbo Medical Treatment Centre Li Huili Hospital https://orcid.org/0000-0001-8684-6485

\section{Primary research}

Keywords: lung adenocarcinoma, long noncoding RNA, tumor immune microenvironment, prognostic signature, survival

Posted Date: June 23rd, 2021

DOl: https://doi.org/10.21203/rs.3.rs-620829/v1

License: (c) (1) This work is licensed under a Creative Commons Attribution 4.0 International License. Read Full License 
Version of Record: A version of this preprint was published at Frontiers in Genetics on December 8th, 2021. See the published version at https://doi.org/10.3389/fgene.2021.767694. 


\section{Abstract}

Background: Autophagy is closely associated with the tumor immune microenvironment (TIME) and prognosis of patients with lung adenocarcinoma (LUAD). In the present study, we established a signature based on long noncoding RNAs (IncRNAs) related to autophagy (ARIncRNAs) to investigate the TIME and survival of LUAD patients.

Methods: We selected ARIncRNAs associated with prognosis to construct a model, and divided each sample into different groups based on risk score. Subsequently, Kaplan-Meier survival analysis was performed to investigate the survival outcomes of LUAD patients in different group. Kyoto Encyclopedia of Genes and Genomes (KEGG) and Gene Ontology (GO) enrichment analysis explored the enriched pathways and functions. Several bioinformatics analyses were conducted to explore the TIME of LUAD patients in different group.

Results: The ARIncRNAs signature could be recognized as an independent prognostic factor for LUAD patients, and patients in the low-risk group had a greater survival advantage. GO and KEGG enrichment analysis suggested that several immune functions and pathways were enriched in different groups. A high-risk score correlated significantly negatively with high abundance of immune cells and stromal cells around the tumor and high tumor mutational burden (TMB). Low-risk patients had a higher PD-1, CTLA-4 and HAVCR2 expression, and had a better efficacy of immune checkpoint inhibitor (ICls), including PD1/CTLA-4 inhibitor.

Conclusions: A reliable signature based on ARIncRNAs was constructed to explore the TIME and prognosis of LUAD patients, which could provide valuable information for individualized LUAD treatment.

\section{Introduction}

Lung cancer is one of the malignant tumors with the highest morbidity and mortality in the world ${ }^{1}$. The incidence and mortality of lung cancer in the United States in 2021 are estimated to be 235,760 and 131,880 , respectively ${ }^{2}$. In China, there were estimated 733,000 new lung cancer cases and 610,000 deaths in $2015^{3}$. Non-small cell lung cancer (NSCLC) accounts for about $85 \%$ of lung cancers, of which adenocarcinoma accounts for about $50 \%$ of NSCLC 4 . Although the advent of radiotherapy and chemotherapy has revolutionized the NSCLC treatment, the 5-year survival rate of NSCLC with distant metastasis is only $7 \%^{5}$. Therefore, it is crucial to screen a reliable biomarker to guide individualized treatment of NSCLC.

As a crucial part of the recycling process in complicated tumor immune microenvironment (TIME), autophagy in tumor immunity has been increasingly appreciated ${ }^{6}$. For example, Jiang et al. illustrated the impact of autophagy on TIME from three perspectives, and proposed that autophagy-based therapy combined with immunotherapy may be promising ${ }^{7}$. Gerada et al. ${ }^{8}$ explained that TIME determines whether autophagy promotes or inhibits tumors. LncRNAs are a class of RNA that do not code proteins 
with transcripts $>200$ nucleotides $^{9}$. They participated in the progression and metastasis of lung adenocarcinoma (LUAD) and were associated with immune pathways, and even served as a biomarker for prognosis of LUAD ${ }^{10}{ }^{11}$. Recently, the prognostic signatures based on coding or non-coding genes to predict the prognosis of LUAD patients has been a research hot spot ${ }^{12}{ }^{13}$. However, utilizing ARIncRNAs to construct models, and exploring the tumor immunity and the efficacy of immunotherapy in LUAD patients was still lacking. In this study, we established a novel ARIncRNAs signature to analyze the TIME and prognosis of LUAD patients, which represented a step toward individualized immunotherapy in LUAD.

\section{Materials And Methods \\ 2.1 Data acquisition}

A signature based on ARIncRNAs was established by a multi-step approach (Fig. 1). We acquired the transcriptome profiles and the corresponding clinical information of LUAD patients and normal samples from The Cancer Genome Atlas (TCGA, https://portal.gdc.cancer.gov/) database in December 2020. The gene transfer format (GTF) files was obtained from Ensembl (http://asia.ensembl.org) to distinguish IncRNAs and mRNAs. Furthermore, a list of autophagy-related genes (ARGs) from the Human Autophagy Database (HADb) (http://www.autophagy.lu/) was acquired. We identified ARIncRNAs by calculating the correlation coefficient between the ARGs and IncRNAs ( $\mid$ cor $\mid>0.4$ and $p<0.001)$.

\subsection{Construction of the ARIncRNAs signature}

Kaplan-Meier survival analysis combined with univariate Cox analysis were performed to screen the ARIncRNAs expression levels that were significantly associated with the overall survival (OS) of the LUAD patients (log-rank $p<0.05$, and $p<0.05$ ). They were chosen for subsequent Cox proportional hazard regression analysis, and a heatmap was plotted for visualization. Subsequently, the multivariate Cox regression analysis was performed to construct the prognostic signature by running the R-x64-4.0.4 survival package, and the risk score of every LUAD patient was calculated based on the following formula:

$\mathrm{n}$

Risk score $=\sum$ Coef $(\mathrm{i}) \times \mathrm{E}(\mathrm{i})$, $i=1$,

Coef (i) and E(i) represent the regression coefficient of the multivariate Cox analysis for each ARIncRNA and each ARIncRNA expression level, respectively. The median value of risk score was considered as the cut-off point for differentiating each patient into different groups.

\subsection{Validation of the risk prognosis model}


Several ROC curves were generated and AUC were calculated by R-x64-4.0.4 survival, survminer, timeROC packages to validate the predictive value of the prognostic signature. Kaplan-Meier log-rank test was conducted for comparing the OS between different risk groups to assess the predictive value of the prognosis model. A barplot and a boxplot was generated by R-x64-4.0.4 packages plyr, ggplot2, ggpubr to study whether there are statistical differences in OS between different risk groups. In addition, univariate and multivariate Cox regression analyses were performed to explore whether the prognostic signature was a potential independent prognostic indicator for patients with LUAD, and the results were visualized with two forest maps. To study the correlation between risk score and clinicopathological characteristics, several chi-square tests were performed to plot boxplots. A heatmap was generated to visualize the expression of ARIncRNAs included in the process of modeling.

\subsection{Enrichment of functions and pathways in the ARIncRNA signature}

Differentially expressed genes between different risk groups were identified by performing differential expression analysis. The R-x64-4.0.4 packages limma was utilized and the significance threshold for determining differentially expressed genes was log fold change $[F C]>1$ and false discovery rate $[F D R]<$ 0.05. To filter functional phenotypes in different risk groups, we performed GSEA 4.0.1 (https://www.gseamsigdb.org/gsea/index.jsp) ${ }^{14}$ by Kyoto Encyclopedia of Genes and Genomes (KEGG) $(p<0.05$ and $q<$ 0.25). GO function enrichment analysis was conducted to study the functional phenotypes that differentially expressed ARIncRNAs were enriched ( $p<0.05$ and $q<0.05$ ).

\subsection{Correlation analysis of tumor mutational burden}

Spearman analysis was conducted to calculate the correlation coefficient between the tumor mutational burden (TMB) and the constructed model based on R-x64-4.0.4 ggplot2, ggpubr and ggExtra packages. The differences in TMB between different risk groups were explored by Wilcoxon signed-rank test, and a column diagram was plotted. To study the connection between TMB and the prognostic signature comprehensively, Kaplan-Meier survival analyses was performed for comparing OS between high-TMB and low-TMB.

\subsection{Estimation of tumor immune microenvironment of the prognostic signature}

To explore the abundance of immune cells and stromal cells between different groups, StromalScore, ImmuneScore, and ESTIMATEScore (StromalScore + ImmuneScore) of each patient were calculated by Rx64-4.0.4 estimate and limma packages. Then, Wilcoxon signed-rank tests were utilized to explore the differences in StromalScore, ImmuneScore, and ESTIMATEScore between different groups, and three column diagrams were plotted for visualization. Single-sample gene-set enrichment analysis (sSGSEA) was conducted for scoring LUAD-infiltrating immune cells to quantify their relative content. The scores of immune cells and pathways in different groups were shown on multi-boxplots, respectively. 


\subsection{Evaluation of clinical immunotherapy efficacy of ARIncRNAs signature}

The emergence of immunotherapy has revolutionized the treatment of NSCLC. National Comprehensive Cancer Network (NCCN) guidelines ${ }^{15}$ recommend a series of immunotherapy drugs, including CTLA-4 and PD-1 blocking antibodies for NSCLC treatment. Expression analyses of common ICl-related immunosuppressive molecules (e.g., PD-1, CTLA-4, and HAVCR-2) were conducted by R-x64-4.0.3, ggpubr and limma packages to detect any statistical differences in expression of common ICl-related immunosuppressive molecules between different risk groups, and several violin plots were generated for visualization. Information of immunotherapy efficacy for LUAD patients was obtained from the The Cancer Immunome Atlas (TCIA, https://tcia.at/) database ${ }^{16}$. The difference in immunotherapy efficacy for LUAD patients between different groups was calculated by Wilcoxon signed-rank tests, and violin plots were labeled as follows for visualization: $* \star \star<0.001$, $* \star<0.01$, and $*<0.05$.

\section{Results}

\subsection{Identification of ARIncRNAs}

Figure1 shows, a multi-step approach to identify ARIncRNAs was conducted. A total of 551 LUAD transcriptome data, corresponding clinical data from TCGA-LUAD cohort (54 normal samples and 497 LUAD samples) and 232 ARGs from HADb were downloaded. Subsequently, we distinguished the mRNAs and IncRNAs by GTF files and identified 1071 ARIncRNAs based on Spearman correlation analysis.

\subsection{Establishment of the prognostic signature based on ARIncRNAs}

Using univariate Cox analysis combined with Kaplan-Meier survival analysis, we screened out 57 ARIncRNAs associated with survival of LUAD patients (Fig. 2a). Multivariate Cox regression analysis yielded 14 ARIncRNAs among ARIncRNAs related to prognosis for subsequent modeling. The risk score of every patient was calculated based on correlation coefficients calculated by multivariate Cox regression analysis, and each sample was differentiated into different groups by median value of risk score.

\subsection{The prognostic signature is a powerful LUAD prognostic indicator}

To quantify the predictive ability of the ARIncRNAs signature, we generated several ROC for validation. The curves demonstrated that $1-, 3$-, and 5-year AUC values were $0.764,0.742$, and 0.713 , respectively (Fig. 2b-c). Furthermore, compared with other clinicopathological characteristics (e.g., age, gender, and stage), the 1-year AUC value was the maximum (Fig. 2d). The survival curve, boxplot, and barplot indicated that patients with low-risk had a better prognosis and were statistically significant (Fig. 3a-C). 
To explore the relationship between the ARIncRNA signature with clinicopathological characteristics, we performed univariate and multivariate Cox regression analysis, which suggested that two factors, i.e., the risk score (hazard ratio $[\mathrm{HR}]=1.589$, confidence interval $[\mathrm{Cl}]=1.406-1.797, p<0.001)$ and stage $(\mathrm{HR}=$ $1.470,[\mathrm{Cl}]=1.265-1.710, p<0.001$ ), correlated with the survival (Fig. 3d-e). The results above suggested that the ARIncRNA signature could act as a potential independent prognostic indicator for patients with LUAD. The heatmap (Fig. 4a) suggested that stage $(p<0.001)$ and ImmuneScore $(p<0.001), \mathrm{N}(p<0.01)$ were statistically different in different groups. In addition, most ARIncRNAs included in the process of modeling were enriched in the low-risk group, suggesting autophagy was more active in the low-risk group. The scatter diagrams indicated that T (Fig. 4b, p<0.01), stage (Fig. 4c, p<0.001), and N (Fig. 4d, $p$ $<0.001$ ) were associated with risk score.

\subsection{Functional annotation of the prognostic signature}

GSEA analysis (Fig. 5a) indicated that several immune pathways such as B cell receptor signaling, natural killer cell mediated cytotoxicity, T cell receptor signaling pathway, and VEGF signaling pathway were enriched in patients with low risk. Cell cycle, P53 signaling pathway, pathways in cancer, small cell lung cancer, and thyroid cancer were relatively more active in the high-risk group. GO function enrichment analysis indicated that several functions related to tumor immunity such as humoral immune response, antimicrobial humoral response, antigen processing and presentation, and T cell mediated cytotoxicity, were enriched in different groups (Fig. 5b).

\subsection{Assessment of the correlation between the TMB and ARIncRNA signature}

The correlation curve (Fig. 5c) and column diagram (Fig. 5d) indicated that TMB was significantly negatively correlated with risk score, and the TMB of patients with high-risk was significantly higher than those with low-risk $(p<0.001)$. According to survival curves (Fig. $5 \mathrm{e}-\mathrm{f})$, patients with high TMB had a better survival advantage $(p=0.035)$, and patients with a combination of low-risk and high TMB showed a great prognosis.

\subsection{Tumor immune microenvironment of the ARIncRNA signature}

We found that StromalScore (Fig. 6a, $p<0.001$ ), ImmuneScore (Fig. 6b, $p<0.001$ ), and ESTIMATEScore (Fig. $6 \mathrm{c}, \mathrm{p}<0.001$ ) in low-risk patients were significantly higher than that of high-risk patients. The multiboxplots (Fig. 6d-e) indicated that the abundance of activated dendritic cells (aDCs), B cells, dendritic cells (DCs), immature dendritic cells (iDCs), mast cells, neutrophils, plasmacytoid dendritic cells (pDCs), $T$ helper cells, tumor infiltrating lymphocytes (TIL), and regulatory $T$ cells (Tregs) associated significantly negatively with the risk score. Compared with the high-risk group, several immune pathways, e.g., clinical complete response (CCR), check-point, cytolytic activity, human leukocyte antigen (HLA), T cell coinhibition, T cell co-stimulation, and type II interferons (type II IFN) response were more active in the lowrisk group. 


\subsection{ARIncRNA signature in the role of immunotherapy}

The column diagrams showed that the expression of common ICl-related immunosuppressive molecules, e.g., PD-1 (Fig. 6f, p < 0.05), CTLA-4 (Fig. 6g, p< 0.001), and HAVCR-2 (Fig. 6h, p< 0.001), in low-risk group were significantly higher than those in high-risk group, which indicated that the efficacy of the abovementioned ICls seem to be better for low-risk patients. The violin plots based on TCIA validated the above results, suggesting that whether it is PD-1 inhibitor alone (Fig. 6i, p<0.05), CTLA-4 inhibitor alone (Fig. 6j, $p<0.001$ ) or a combination of the two (Fig. $6 k, p<0.001$ ), the efficacy of low-risk patients is better than that of high-risk patients.

\section{Discussion}

Although there are already lots of signatures utilizing ARGs or ARIncRNAs to predict LUAD patients' survival outcomes, we are the first to explore the tumor immunity of the ARIncRNA model in detail ${ }^{17} 18$. We conducted a detailed study on the tumor immunity of the ARIncRNA signature, which renders the constructed signature applicable for guiding the clinical personalized treatment of LUAD patients.

First, the IncRNA and ARG transcriptome profiles were obtained, and ARIncRNAs related to prognosis of LUAD patients based on co-expression analysis and univariate Cox analysis were identified. Next, we calculated the AUC value of 1-, 3-, and 5-year to obtain an ideal signature and differentiated each patient into different groups based on median value. Then, the survival and clinicopathological characteristics was analyzed to assess the predictive value of the ARIncRNA signature. Subsequently, we conducted a comprehensive assessment of the tumor immunity of the ARIncRNA signature, including GO and KEGG function enrichment analyses, TMB, TME, infiltration of immune cells, expression of common ICl-related immunosuppressive molecules, and efficacy of ICls.

Previous studies on LUAD have mostly focused on single genes or noncoding genes, which are unable to illustrate the complex tumorigenesis and development process ${ }^{19}{ }^{20}$. In recent years, a combination of several genes to improve the predictive value of OS in LUAD patients were gradually identified. For example, Duan et al. ${ }^{18}$ constructed a prognostic signature based on ARGs, which served as a novel biomarker in LUAD. Meanwhile, Zhu et al. ${ }^{21}$ identified a ferroptosis-related gene signature and explored the immune cells infiltration. In this study, several ARIncRNAs included in the modeling process have been already reported in various malignant tumors, such as CARD8-AS122 23, AC060780.124, AC123595.1 2526 , UGDH-AS1 ${ }^{27}$, LINC00996 ${ }^{28}$, LINC00861 293031 , AL606489.1 17, HLA-DQB1-AS1 ${ }^{32}$, LINC00654 ${ }^{33}$ LINC00847 34 . While others have not been discovered yet and may be potential novel biomarkers for further study.

Researchers found that autophagy played a vital role in tumorigenesis and development. Gu et al. confirmed that bupivacaine induced autophagy through Akt/mTOR signaling, inhibiting the progression of NSCLC ${ }^{36}$. Lin et al. proposed that high expression of miR-30a improved the prognosis of NSCLC after 
neoadjuvant chemotherapy by reducing autophagy caused by chemotherapy drugs ${ }^{37}$. In addition, Li et al. found that the dysfunction of autophagy mediated by c-myc/miR-150/EPG5 had a great impact on the progression of $\mathrm{NSCLC}^{38}$. Collectively, autophagy was probably involved in the occurrence and development of LUAD through a certain signaling pathway, having a significant impact on the prognosis of LUAD patients.

Then, GSEA enrichment analysis suggested that patients with low-risk had more autophagy and were enriched in B cell receptor signaling, natural killer cell mediated cytotoxicity, T cell receptor signaling pathway, and the VEGF signaling pathway. Recent searches confirmed the strong correlation between autophagy and VEGF. For example, Chen et al. found that VEGF promoted the occurrence of autophagy and VEGF knockdown decreased the autophagy level ${ }^{39}$. In addition, Spengler et al. ${ }^{40}$ discovered that VEGF signaling pathway regulated autophagy in endothelial cells. Taken together, we speculated that autophagy probably contributed to the occurrence and development of LUAD through VEGF signaling pathway, and that the ARIncRNA signature was closely associated with tumor immunity.

Recently, the interaction between autophagy and tumor immunity was investigated comprehensively. For example, TMB and TIME were identified as important determinants of the efficacy of ICls and in the prognosis of cancer patients ${ }^{41}$. Moreover, Jena et al. ${ }^{42}$ have demonstrated that autophagy was closely related to TIME and participated in tumor progression. To explore the relationship between the ARIncRNA signature and tumor immunity thoroughly, we conducted sSGSEA to investigate the immune status in different groups. The patients in low-risk group had a higher abundance of immune cells and were more active in immune pathways, most of which were validated closely associated with autophagy. For example, Di et al. ${ }^{43}$ found that CALCOCO2, an autophagy receptor, was mainly expressed in B cells, which mediated autophagy. In addition, the function of DCs to secrete cytokines has been shown to be inhibited by autophagy ${ }^{44}$. Turan et al. ${ }^{45}$ proved through experiments that iDCs infected with TSV-1 could induce autophagy. Li et al. ${ }^{46}$ demonstrated that autophagy of mast cells could serve as a therapeutic target for allergic reactions. Ding et al. ${ }^{47}$ revealed that neutrophils were associated with autophagy. Autophagy has been validated to fuel $\mathrm{pDCs}^{48}$. Schmid et al. ${ }^{49}$ proposed that CD4(+) T helper cells could recognize MHCII molecules presented after autophagy. Lu et al. ${ }^{50}$ demonstrated that autophagy could mediate the function of Tregs. Samuel et al. ${ }^{51}$ proved that cellular metabolism facilitated autophagy to mediate the cytolytic effect. Zhang et al. ${ }^{52}$ confirmed the firm correlation between autophagy and HLA. These studies above suggest that autophagy is closely linked to tumor immunity, and autophagy probably participates in LUAD progression by regulating tumor immunity.

At present, PD-1 ${ }^{53}$ and CTLA-4 ${ }^{54}$ inhibitors have been validated to benefit patients with advanced NSCLC in clinical trials. Furthermore, research indicated that the autophagy of tumor cells increased the expression of ICl-related immunosuppressive molecules (e.g., PD-1 and CTLA-4), and affected anti-tumor immune responses directly. In this study, expression analyses was conducted to study the correlation between the ARIncRNA signature and the expression of common ICl-related immunosuppressive molecules, which revealed low-risk patients always had a higher expression of them and a better efficacy 
of ICls. Subsequently, we analyzed the efficacy of ICls and verified the results above; demonstrating that regardless of whether it is a PD- 1 inhibitor alone, a CTLA-4 inhibitor alone or a combination of the two, the efficacy of patients in the low-risk group is better than that of the high-risk patients. Overall, the ARIncRNA signature could serve as a novel indicator for screening patients applicable for ICls.

According to our data, we speculated that compared with high-risk patients, low-risk patients have more active autophagy, stronger tumor immunity, greater survival advantage and are more applicable for ICls treatment. Autophagy could play an crucial part in the progression of LUAD by regulating the tumor immunity through VEGF signaling pathway, which had a great impact on prognosis of LUAD patients.

However, there are several limitations in our research. Firstly, bias of the information in the analysis process, as the profiles were obtained from public database. Secondly, it was difficult to find an ideal Gene Expression Omnibus (GEO) set including both 14 IncRNAs newly identified and detailed clinical information to validate the constructed ARIncRNA signature. Finally, external validation, such as quantitative real-time PCR and microarrays are necessary to increase credibility.

In conclusion, we constructed a novel ARIncRNAs signature and predicted the survival of LUAD patients, the state of TIME, and even the efficacy of ICls accurately based on the expression of the 14 ARIncRNAs included in the modeling process, which may benefit patients with advanced NSCLC. Immunotherapy combined with TIME targeted therapy may improve individualized treatment of LUAD in the future.

\section{Declarations}

\section{Ethics approval and consent to participate}

Not applicable

\section{Consent for publication}

Written informed consent for publication was obtained from all participants.

\section{Availability of data and materials}

All data analysed during the current study are accessible from the TCGA database(https://portal.gdc.cancer.gov/).

\section{Competing interests}

The authors declare that they have no conflicts of interest. 


\section{Funding}

The study was funded by Basic public welfare project of Ningbo (2019C50041), Zhejiang Province Medical and Health Project (Grant No. 2019319634), The Natural Science Foundation of Ningbo (Grant No. 202003N4273), Zhejiang Province Medical and Health Project (Grant No. 2019KY606), and Nature Science Foundation of Ningbo city (Grant No.2019A610230).

\section{Authors' contributions}

Hang Chen and Guodong Xu contributed to the conception of the study;

Menglu Sang and Saiqi Ni performed the R language;

Yao Lin, Chengfang $\mathrm{Wu}$, and Yinyu Mu contributed significantly to analysis and manuscript preparation; Hang Chen performed the data analyses and wrote the manuscript;

Kaitai Liu, Shibo Wu, and Ni Li helped perform the analysis with constructive discussions.

\section{Acknowledgements}

We thank the TCGA database for generously sharing a large amount of data.

\section{References}

1. Nasim, F., Sabath, B., Eapen, G. A. (2019). Lung cancer. The Medical Clinics of North America, 103, 463-73. https://doi.org/10.1016/j.mcna.2018.12.006

2. Siegel, R., Miller, K., Fuchs, H., Jemal, A. (2021). Cancer statistics, 2021. CA: A Cancer Journal for Clinicians, 71, 7-33. https://doi.org/10.1016/j.ejca.2018.07.005

3. Chen, W., Zheng, R., Baade, P., Zhang, S., Zeng, H., Bray, F., Jemal, A., Yu, X., He, J. (2016). Cancer statistics in China, 2015. CA: A Cancer Journal for Clinicians, 66, 115-32. https://doi.org/10.3322/caac.21338

4. Shi, J., Wang, L., Wu, N., et al. (2018). Clinical characteristics and medical service utilization of lung cancer in China, 2005-2014: Overall design and results from a multicenter retrospective epidemiologic survey. Lung cancer, 128, 91-100. https://doi.org/10.1016/j.lungcan.2018.11.031

5. American Cancer Society. Non-small cell lung cancer survival rates by stage[OL]. www.cancer.org/cancer/non-small-cell-lung-cancer/detection-diagnosis-staging/survivalrates.html.Date last updated: 04/20/21.

6. Li, Y., Lam, S., Zheng, C., Ho, J. (2015). The Effect of Tumor Microenvironment on Autophagy and Sensitivity to Targeted Therapy in EGFR-Mutated Lung Adenocarcinoma. Journal of Cancer, 6, 382-6. https://doi.org/10.7150/jca.11187 
7. Jiang, T., Chen, X., Ren, X., Yang, J., Cheng, Y. (2021). Emerging role of autophagy in anti-tumor immunity: Implications for the modulation of immunotherapy resistance. Drug resistance updates, 56, 100752. https://doi.org/10.1016/j.drup.2021.100752

8. Gerada, C., Ryan, K. (2021). Autophagy, the innate immune response and cancer. Molecular oncology, 14, 1913-29. https://doi.org/10.1002/1878-0261.12774

9. Cai, P., Otten, A., Cheng, B., et al. (2020). PRANCRA genome-wide long noncoding RNA CRISPRi screen identifies as a novel regulator of epidermal homeostasis. Genome research, 30, 22-34. https://doi.org/10.1101/gr.251561.119

10. Wei, Y., Yan, Z., Wu, C., et al. (2017). Integrated analysis of dosage effect IncRNAs in lung adenocarcinoma based on comprehensive network. Oncotarget, 8, 71430-46. https://doi.org/10.18632/oncotarget.19864

11. Pang, Z., Chen, X., Wang, Y., et al. (2021). Long non-coding RNA C5orf64 is a potential indicator for tumor microenvironment and mutation pattern remodeling in lung adenocarcinoma. Genomics, 113, 291-304. https://doi.org/10.1016/j.ygeno.2020.12.010

12. Zhang, B., Wang, R., Li, K., et al. (2021). An Immune-Related IncRNA Expression Profile to Improve Prognosis Prediction for Lung Adenocarcinoma: From Bioinformatics to Clinical Word. Frontiers in oncology, 11, 671341. https://doi.org/10.3389/fonc.2021.671341

13. Li, Y., Shen, R., Wang, A., et al. (2021). Construction of a Prognostic Immune-Related LncRNA Risk Model for Lung Adenocarcinoma. Frontiers in cell and developmental biology, 9, 648806. https://doi.org/10.3389/fcell.2021.648806

14. Subramanian, A., Tamayo, P., Mootha, V., et al. (2005). Gene set enrichment analysis: a knowledgebased approach for interpreting genome-wide expression profiles. Proceedings of the National Academy of Sciences of the United States of America, 102, 15545-50. https://doi.org/10.1073/pnas.0506580102

15. National Comprehensive Cancer Network: NCCN clinical practice guidelines in oncology: Non-Small Cell Lung Cancer, V.1.2021. https://www.nccn.org/login? ReturnURL=https://www.nccn.org/professionals/physician_gls/pdf/nscl.pdf.

16. Hugo, W., Zaretsky, J., Sun, L., et al. (2017). Genomic and Transcriptomic Features of Response to Anti-PD-1 Therapy in Metastatic Melanoma. Cell, 168, 542. https://doi.org/10.1016/j.cell.2017.01.010

17. Wu, L., Wen, Z., Song, Y., Wang, L. (2021). A novel autophagy-related IncRNA survival model for lung adenocarcinoma. Journal of cellular and molecular medicine. https://doi.org/10.1111/jcmm.16582

18. Duan, J., Lei, Y., Lv, G., et al. (2021). Identification of a novel autophagy signature for predicting survival in patients with lung adenocarcinoma. PeerJ, 9: e11074. https://doi.org/10.7717/peerj.11074

19. Wang, W., Li, A., Han, X., et al. (2020). DEPDC1 up-regulates RAS expression to inhibit autophagy in lung adenocarcinoma cells. Journal of cellular and molecular medicine, 24, 13303-13. https://doi.org/10.1111/jcmm.15947 
20. Fan, X., Weng, Y., Bai, Y., et al. (2018). Lipin-1 determines lung cancer cell survival and chemotherapy sensitivity by regulation of endoplasmic reticulum homeostasis and autophagy. Cancer medicine, 7 , 2541-54. https://doi.org/10.1002/cam4.1483

21. Zhu, G., Huang, H., Xu, S., et al. (2021). Prognostic value of ferroptosis-related genes in patients with lung adenocarcinoma. Thoracic cancer. https://doi.org/10.1111/1759-7714.13998

22. Lin, X., Jiang, T., Bai, J., et al. (2018). Characterization of Transcriptome Transition Associates Long Noncoding RNAs with Glioma Progression. Molecular therapy Nucleic acids, 13, 620-32. https://doi.org/10.1016/j.omtn.2018.10.009

23. Li, N., Zhan, X. (2019). Identification of clinical trait-related IncRNA and mRNA biomarkers with weighted gene co-expression network analysis as useful tool for personalized medicine in ovarian cancer. The EPMA journal, 10, 273-90. https://doi.org/10.1007/s13167-019-00175-0

24. Curty, G., Beckerle, G., Iñiguez, L., et al. (2020). Human Endogenous Retrovirus Expression Is Upregulated in the Breast Cancer Microenvironment of HIV Infected Women: A Pilot Study. Frontiers in oncology, 10, 553983. https://doi.org/10.3389/fonc. 2020.553983

25. Li, J., Li, R., Liu, X., et al. (2020). A Seven Immune-Related IncRNAs Model to Increase the Predicted Value of Lung Adenocarcinoma. Frontiers in oncology, 10, 560779. https://doi.org/10.3389/fonc.2020.560779

26. Zheng, H., Li, B., Liu, C., Jia, L., Liu, F. (2020). Comprehensive Analysis of IncRNA-Mediated ceRNA Crosstalk and Identification of Prognostic Biomarkers in Wilms' Tumor. BioMed research international, 2020, 4951692. https://doi.org/10.1155/2020/4951692

27. Liu, H., Zhang, Q., Lou, Q., et al. (2020). Differential Analysis of IncRNA, miRNA and mRNA Expression Profiles and the Prognostic Value of IncRNA in Esophageal Cancer. Pathology oncology research, 26, 1029-39. https://doi.org/10.1007/s12253-019-00655-8

28. Ge, H., Yan, Y., Wu, D., Huang, Y., Tian, F. (2018). Potential role of LINC00996 in colorectal cancer: a study based on data mining and bioinformatics. OncoTargets and therapy, 11, 4845-55. https://doi.org/10.2147/OTT.S173225

29. Hu, W., Wang, Y., Fang, Z., He, W., Li, S. (2021). Integrated Characterization of IncRNA-Immune Interactions in Prostate Cancer. Frontiers in cell and developmental biology, 9, 641891. https://doi.org/10.3389/fcell.2021.641891

30. Liu, H., Zhang, L., Ding, X., Sui, X. (2021). LINC00861 inhibits the progression of cervical cancer cells by functioning as a ceRNA for miR-513b-5p and regulating the PTEN/AKT/mTOR signaling pathway. Molecular medicine reports, 23. https://doi.org/10.3892/mmr.2020.11662

31. Qian, Y., Mao, Z., Shi, Y., Liu, Z., Cao, Q., Zhang, Q. (2018). Comprehensive Analysis of miRNA-mRNAIncRNA Networks in Non-Smoking and Smoking Patients with Chronic Obstructive Pulmonary Disease. Cellular physiology and biochemistry, 50, 1140-53. https://doi.org/10.1159/000494541

32. Singh, A., Gasman, B. (2020). Disentangling the genetics of sarcopenia: prioritization of NUDT3 and KLF5 as genes for lean mass \& HLA-DQB1-AS1 for hand grip strength with the associated enhancing SNPs \& a scoring system. BMC medical genetics, 21, 40. https://doi.org/10.1186/s12881-020-0977-6 
33. Li, L., Gan, Z., Qin, L., Jiao, S., Shi, Y. (2017). AlB1 regulates the ovarian cancer cell cycle through TUG1. European review for medical and pharmacological sciences, 21, 5610-7. https://doi.org/10.26355/eurrev_201712_14002

34. Li, H., Chen, Y., Wan, Q., et al. (2021). Long Non-coding RNA LINC00847 Induced by E2F1 Accelerates Non-small Cell Lung Cancer Progression Through Targeting miR-147a/IFITM1 Axis. Frontiers in medicine, 8, 663558. https://doi.org/10.3389/fmed.2021.663558

35. Tu, L., Li, W., Liu, J., Song, X., Xu, H. (2020). LncRNA LINC00847 contributes to hepatocellular carcinoma progression by acting as a sponge of miR-99a to induce E2F2 expression. Journal of biological regulators and homeostatic agents, 34, 2195-203. https://doi.org/10.23812/20-427-L

36. Gu, J., Liu, C., Xie, J., et al. (2021). The Local Anesthetic Bupivacaine Inhibits the Progression of NonSmall Cell Lung Cancer by Inducing Autophagy Through Akt/mTOR Signaling. Frontiers in oncology, 11, 616445. https://doi.org/10.3389/fonc.2021.616445

37. Lin, X., Lai, X., Feng, W., Yu, X., Gu, Q., Zheng, X. (2021). MiR-30a sensitized lung cancer against neoadjuvant chemotherapy by depressing autophagy. Japanese journal of clinical oncology, 51, 67584. https://doi.org/10.1093/jjco/hyaa272

38. Li, H., Liu, J., Cao, W., et al. (2019). C-myc/miR-150/EPG5 axis mediated dysfunction of autophagy promotes development of non-small cell lung cancer. Theranostics, 9, 5134-48. https://doi.org/10.7150/thno.34887

39. Chen, L., Lin, G., Chen, K., et al. (2020). VEGF knockdown enhances radiosensitivity of nasopharyngeal carcinoma by inhibiting autophagy through the activation of mTOR pathway. Scientific reports, 10, 16328. https://doi.org/10.1038/s41598-020-73310-x

40. Spengler, K., Kryeziu, N., Große, S., Mosig, A., Heller, R. (2020). VEGF Triggers Transient Induction of Autophagy in Endothelial Cells via AMPKa1. Cells, 9. https://doi.org/10.3390/cells9030687

41. Wu, J., Xu, C., Guan, X., et al. (2021). Comprehensive analysis of tumor microenvironment and identification of an immune signature to predict the prognosis and immunotherapeutic response in lung squamous cell carcinoma. Annals of translational medicine, 9, 569. https://doi.org/10.21037/atm-21-463

42. Jena, B., Rout, L., Dey, A., Mandal, M. (2021). Active autophagy in cancer-associated fibroblasts: Recent advances in understanding the novel mechanism of tumor progression and therapeutic response. Journal of cellular physiology. https://doi.org/10.1002/jcp.30419

43. Di Rita, A., Strappazzon, F. (2021). A protective variant of the autophagy receptor CALCOCO2/NDP52 in Multiple Sclerosis (MS). Autophagy, 1-3. https://doi.org/10.1080/15548627.2021.1924969

44. Liang, L., Zhou, Q., Feng, L. (2021). Decreased microRNA-155 in Behcet's disease leads to defective control of autophagy thereby stimulating excessive proinflammatory cytokine production. Arthritis research \& therapy, 23, 135. https://doi.org/10.1186/s13075-021-02517-8

45. Turan, A., Grosche, L., Krawczyk, A., et al. (2019). Autophagic degradation of lamins facilitates the nuclear egress of herpes simplex virus type 1 . The Journal of cell biology, 218, 508-23. https://doi.org/10.1083/jcb.201801151 
46. Li, J., Ullah, M., Jin, H., et al. (2021). viaORMDL3 Functions as a Negative Regulator of AntigenMediated Mast Cell Activation an ATF6-UPR-Autophagy-Dependent Pathway. Frontiers in immunology, 12, 604974. https://doi.org/10.3389/fimmu.2021.604974

47. Ding, L., Yang, J., Zhang, C., Zhang, X., Gao, P. (2021). Neutrophils Modulate Fibrogenesis in Chronic Pulmonary Diseases. Frontiers in medicine, 8, 616200. https://doi.org/10.3389/fmed.2021.616200

48. Basit, F., Mathan, T., Sancho, D., de Vries, I. (2021). Human Dendritic Cell Subsets Undergo Distinct Metabolic Reprogramming for Immune Response. Frontiers in immunology, 9, 2489. https://doi.org/10.3389/fimmu.2018.02489

49. Schmid, D., Dengjel, J., Schoor, O., Stevanovic, S., Münz, C. (2006). Autophagy in innate and adaptive immunity against intracellular pathogens. Journal of molecular medicine, 84, 194-202. https://doi.org/10.1007/s00109-005-0014-4

50. Lu, J., Liu, Y., Wang, W., Li, P., Qi, F. (2021). Knockdown of miR-146a in regulatory T cells suppresses heart transplantation rejection in mice by increasing autophagy. Transplant immunology, 65, 101372. https://doi.org/10.1016/j.trim.2021.101372

51. Samuel, S., Beljanski, V., Van Grevenynghe, J., et al. (2013). BCL-2 inhibitors sensitize therapyresistant chronic lymphocytic leukemia cells to VSV oncolysis. Molecular therapy, 21, 1413-23. https://doi.org/10.1038/mt.2013.91

52. Zhang, X., Jin, Y., Wang, Q., et al. (2020). Autophagy-mediated regulation patterns contribute to the alterations of the immune microenvironment in periodontitis. Aging, 13, 555-77. https://doi.org/10.18632/aging.202165

53. Fan, T., Liu, Y., Liu, H., et al. (2021). Comprehensive analysis of a chemokine- and chemokine receptor family-based signature for patients with lung adenocarcinoma. Cancer immunology, immunotherapy, Cll 2021. https://doi.org/10.1007/s00262-021-02944-1

54. Sun, T., Zhang, W., Li, Y., et al. (2020). Combination Immunotherapy with Cytotoxic T-LymphocyteAssociated Antigen-4 and Programmed Death Protein-1 Inhibitors Prevents Postoperative Breast Tumor Recurrence and Metastasis. Molecular cancer therapeutics, 19, 802-11. https://doi.org/10.1158/1535-7163.MCT-19-0495

\section{Figures}




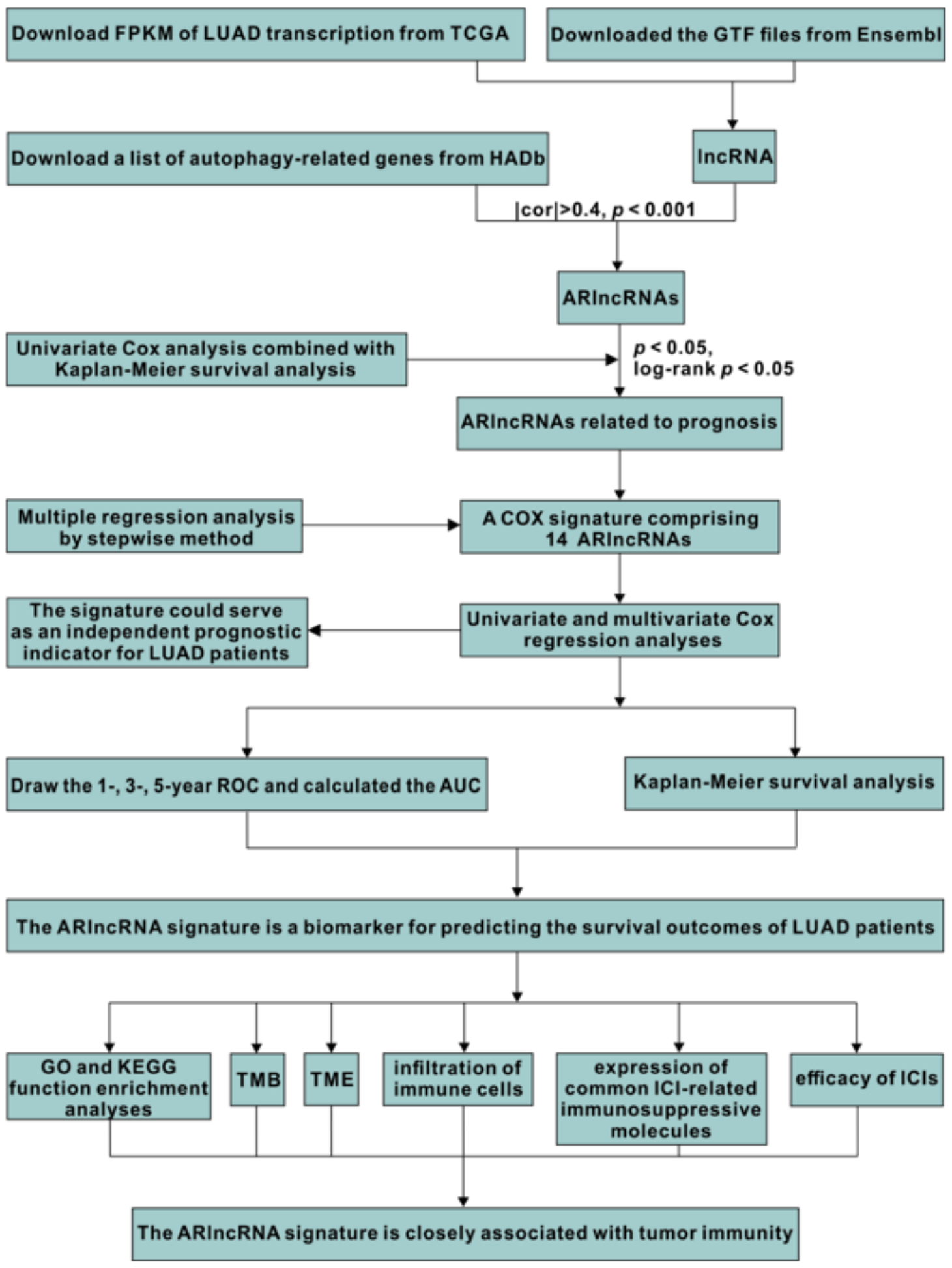

\section{Figure 1}

The flowchart for identifying a novel reliable ARIncRNAs signature and its implication. 
a
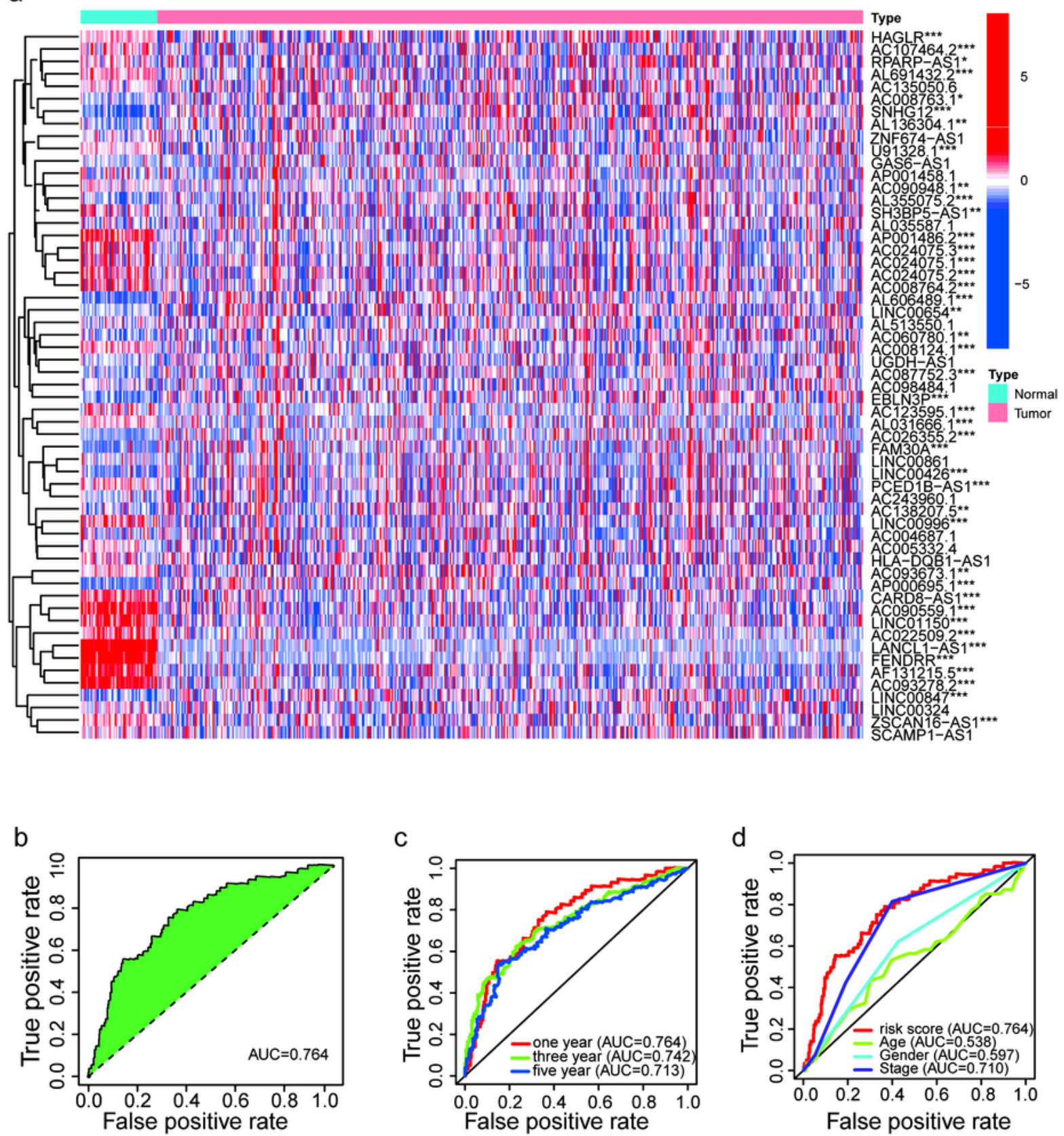

\section{Figure 2}

Identification of the ARIncRNA signature. (a) Heatmap shows the expression of ARIncRNAs related to survival in LUAD and normal samples. (b and c) The 1-, 3-, 5-year AUC values were >0.7. (d) Compared with AUC of age, sex, and stage, the 1-year AUC value was the maximum. 


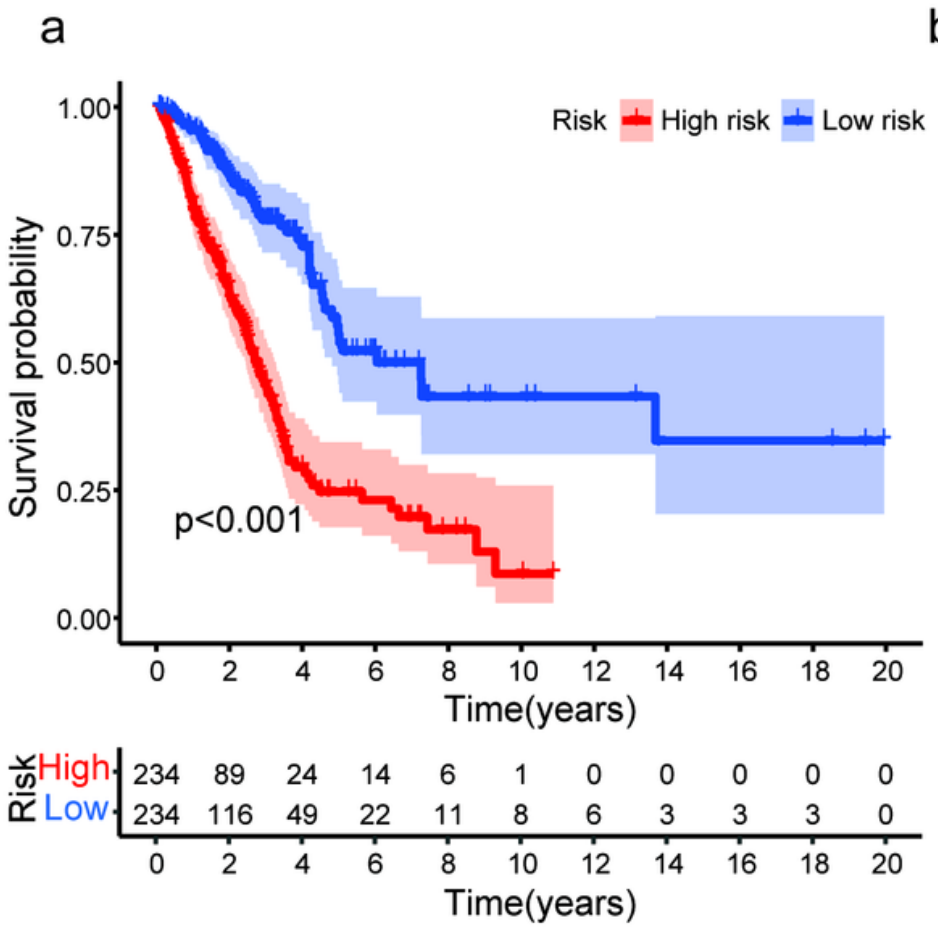

b

C

Fustat \Alive】Dead
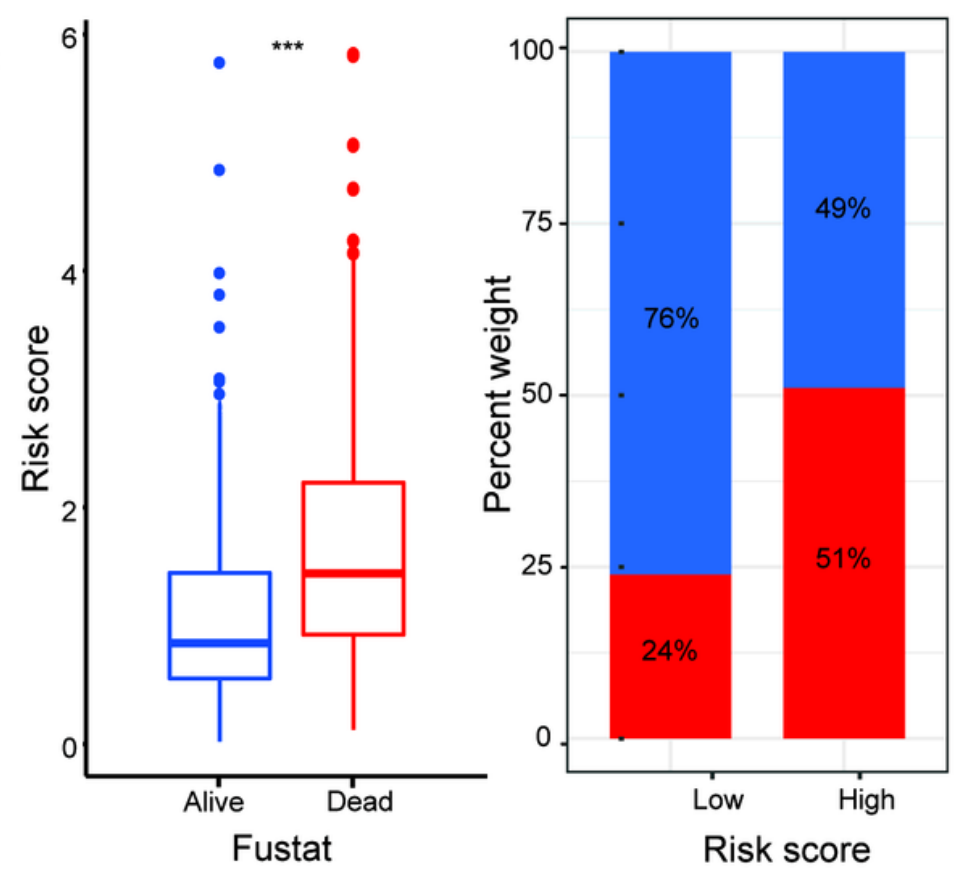

d

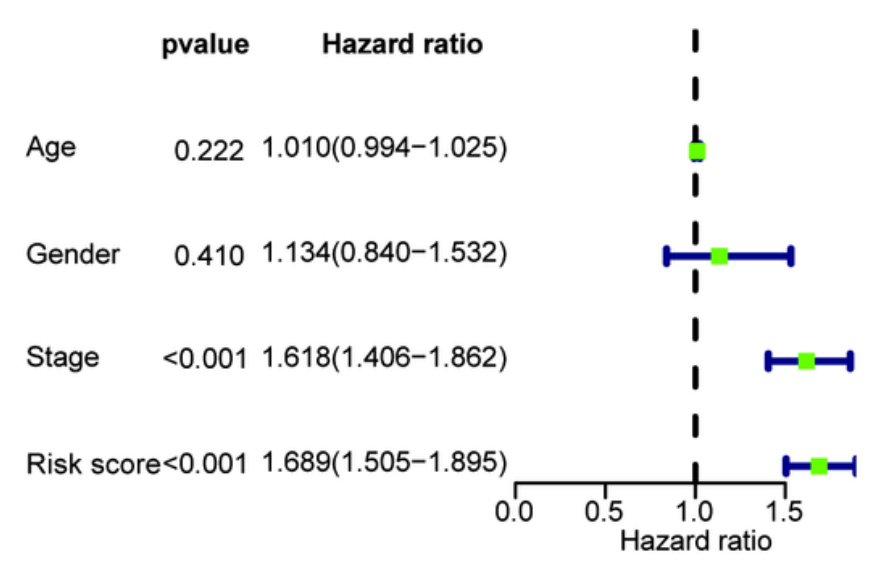

e

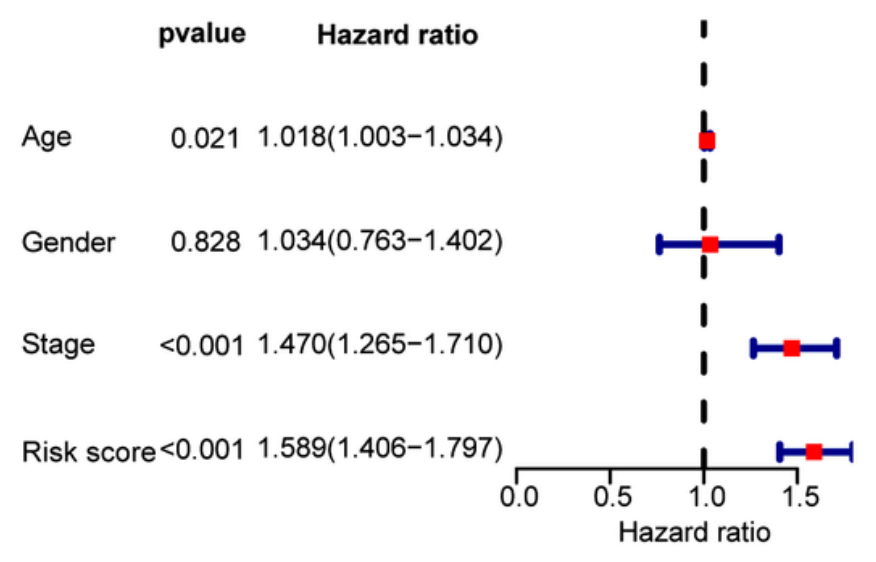

\section{Figure 3}

Validation of the predictive value of the prognostic signature. (a-c) The survival curve (a), boxplot (b), and barplot (c) indicate that the ARIncRNA signature was associated with survival outcomes of LUAD patients and patients with low risk have a significant survival advantage. (d and e) Univariate (d) and multivariate (e) Cox regression analyses including age, gender, stage, risk score suggests that risk score $(H R=1.589$, $\mathrm{Cl}=1.406-1.797, \mathrm{p}<0.001)$ and stage $(\mathrm{HR}=1.470, \mathrm{Cl}=1.265-1.710, \mathrm{p}<0.001)$ could act as an independent prognostic factor for LUAD. 
a
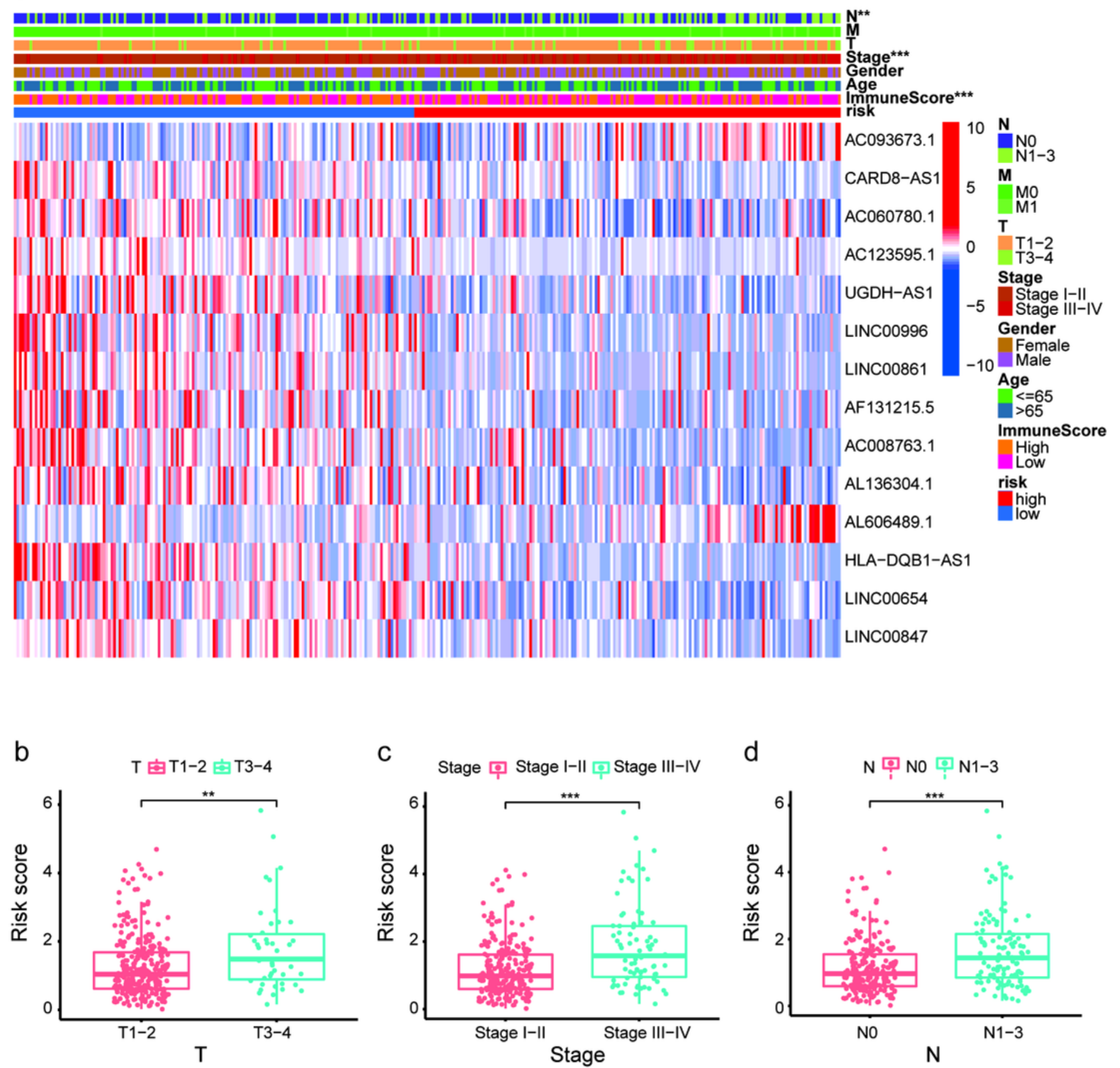

C Stage 무 Stage I-II 무 Stage III-IV

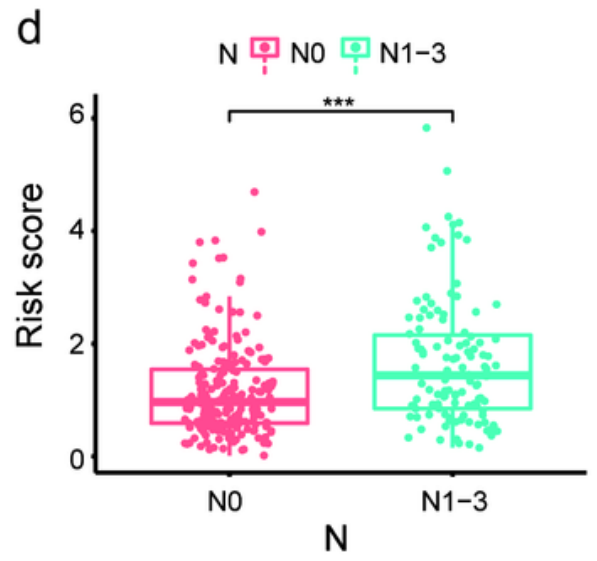

Figure 4

Exploration of clinicopathological characteristics of the 14-ARIncRNA signature. (a) The heatmap suggested that stage $(p<0.001)$, ImmuneScore $(p<0.001)$, and $N(p<0.01)$ were statistically different in different groups. In addition, most ARIncRNAs included in the modeling process were enriched in the lowrisk group, suggesting autophagy was more active in the low-risk group. (b-d) The scatter diagrams indicate that higher levels of T (b), N (c), and stage (d) tend to have a higher risk score. 
a

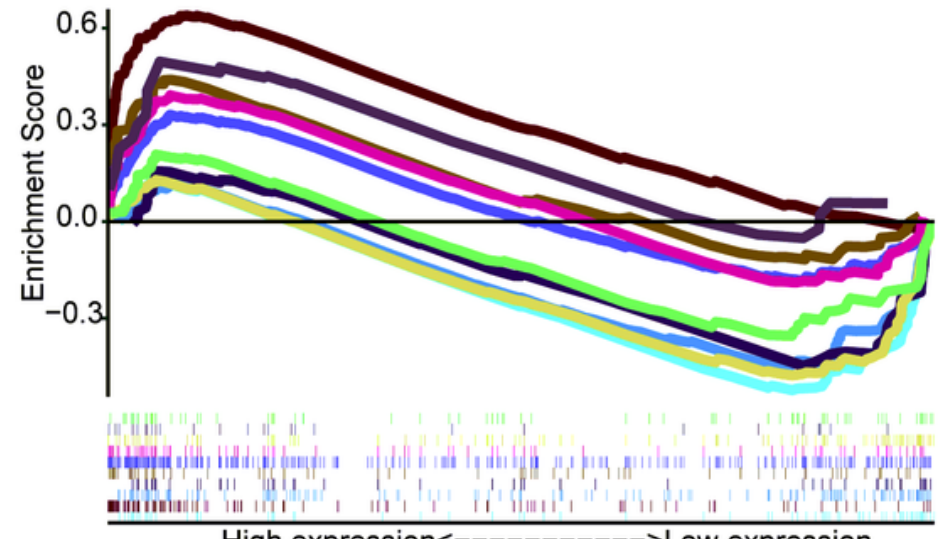

High expression<------------>Low expression

b

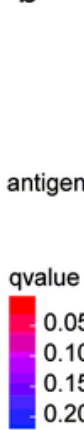
antimicrobial immune response antimicrobial humoral response formation of quadruple SL/U4/U5/U6 snRNP mRNA trans splicing, via spliceosome
mRNA trans splicing. SL addition mRNA trans splicing, SL addition T cell mediated cytotoxicity
spliceosomal tri-snRNP complex assembly antigen processing and presentation via MHC class Ib regulation of $\mathrm{T}$ cell mediated cytotoxicity

external side of plasma membrane U4/U6 x U5 tri-snRNP complex
U spliceosomal tri-snRNP complex tertiary granule lumen nuclear envelope lumen anchored component of plasma membrane
spliceosomal snRNP complex collagen-containing extracellular matrix small nuclear ribonucleoprotein complex. laminin complex

lipopeptide binding
immunoglobulin binding
growth factor activity . receptor ligand activity signaling receptor activator activity serine-type peptidase activity serine hydrolase activity DNA endonuclease activity

e
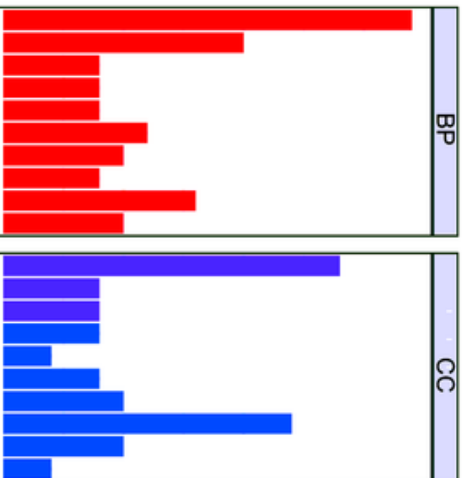

C

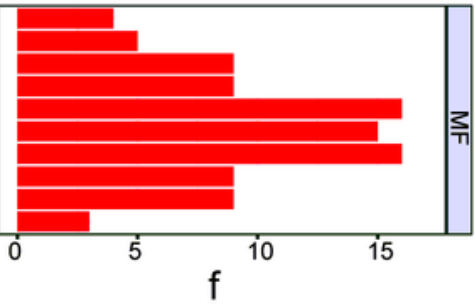

$\mathrm{d}$
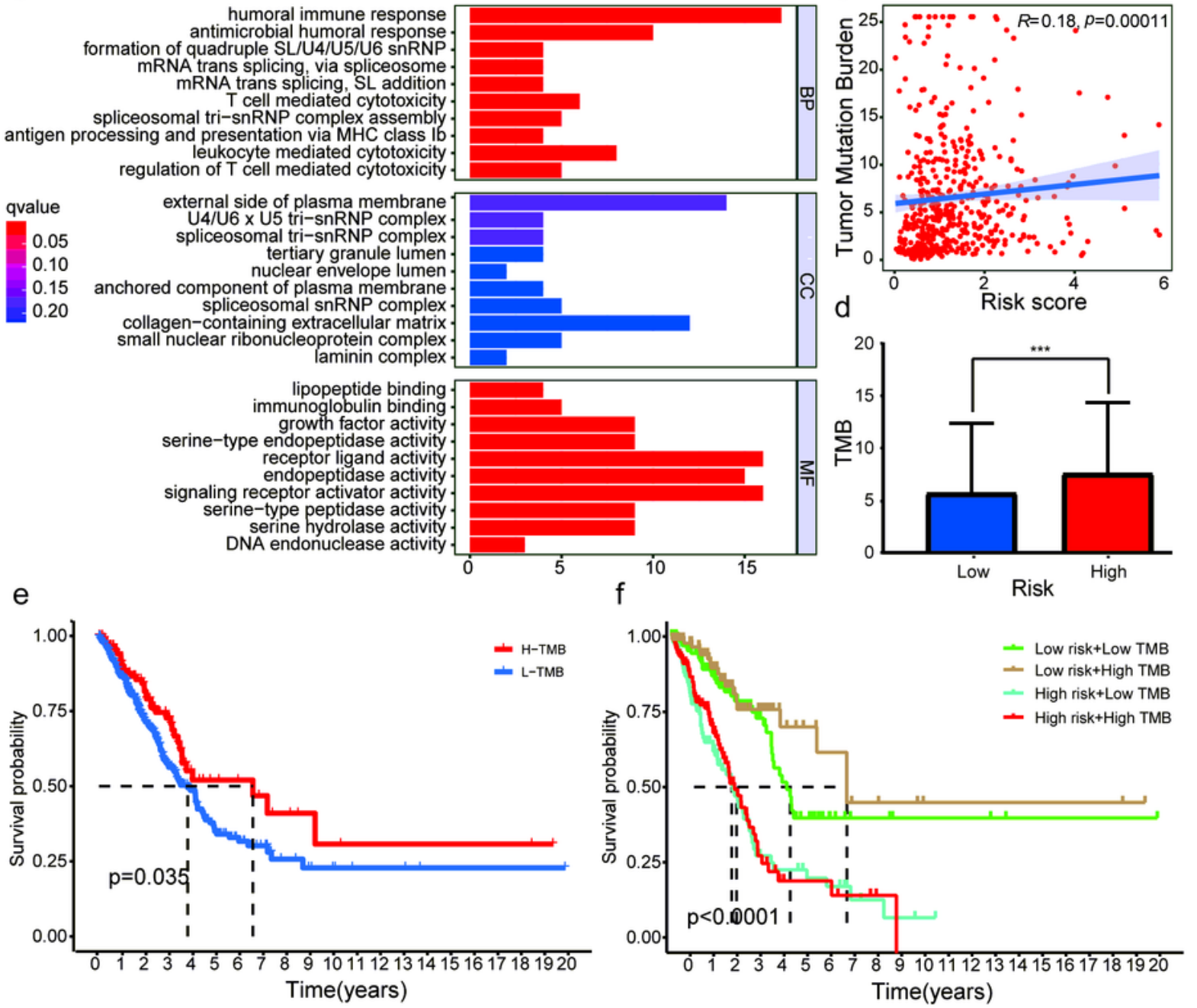

Figure 5

Research on tumor immunity of the 14-ARIncRNA signature. ( $a$ and b) KEGG (a) and GO (b) function enrichment analyses reveal that several immune pathways and immune function were enriched in different groups, suggesting the ARIncRNA signature may be associated with tumor immunity. (c and d) The scatter diagram (c) and column diagram (d) show that high-risk patients exhibit significantly higher 
TMB. (e and f) The survival curves indicate that patients with a combination of low-risk and high TMB showed a greater prognosis.

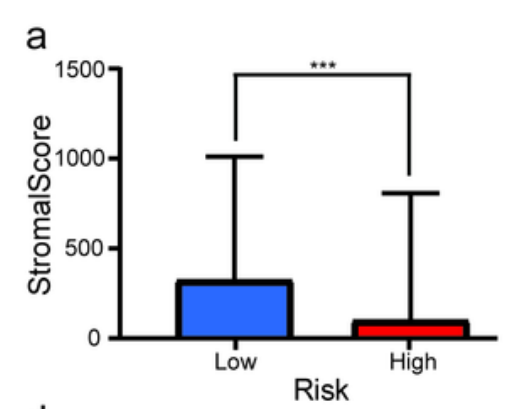

d

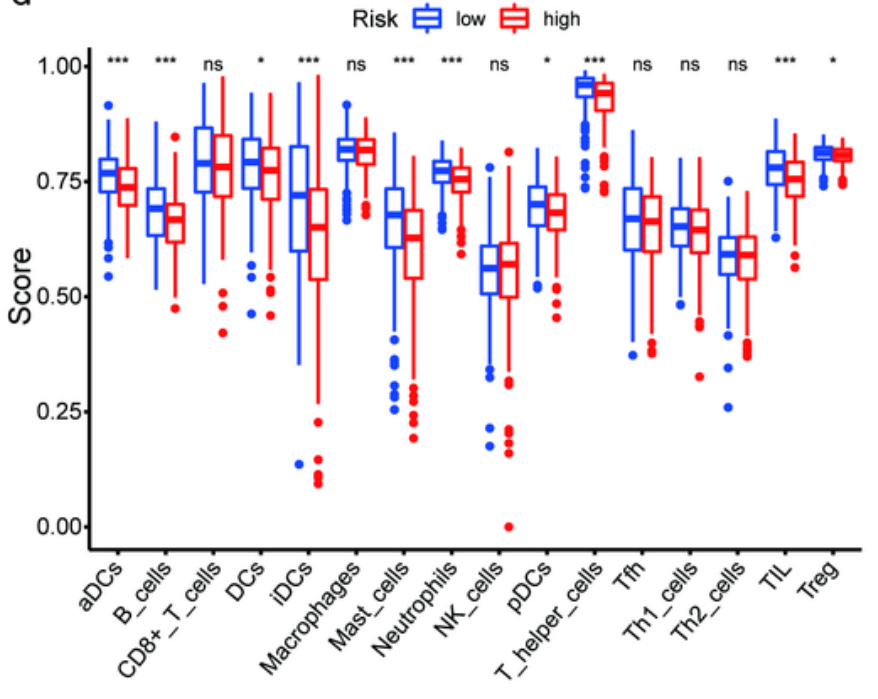

f
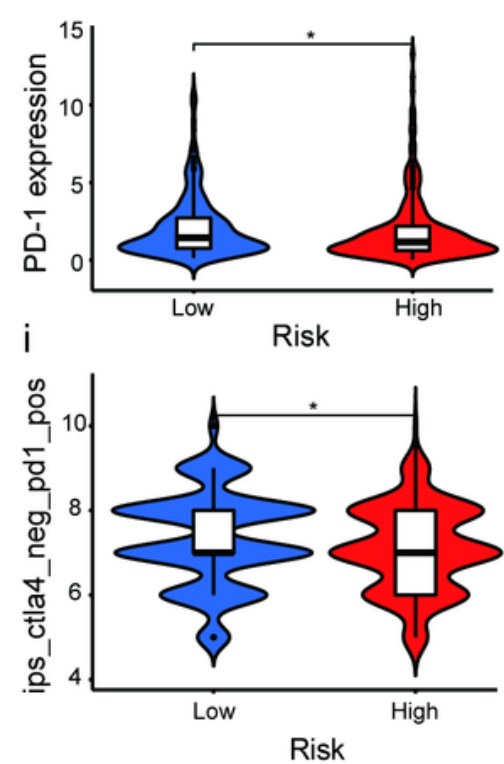

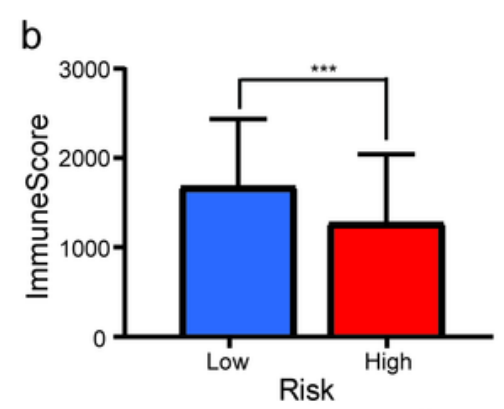

e

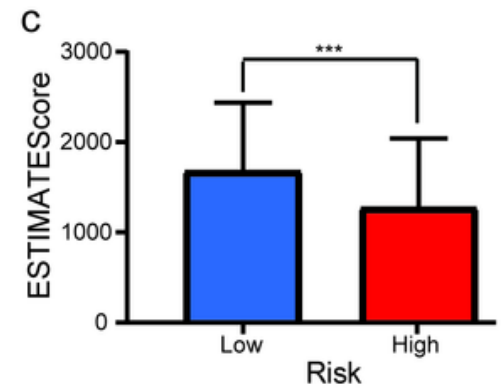

Risk 追 low 追 high
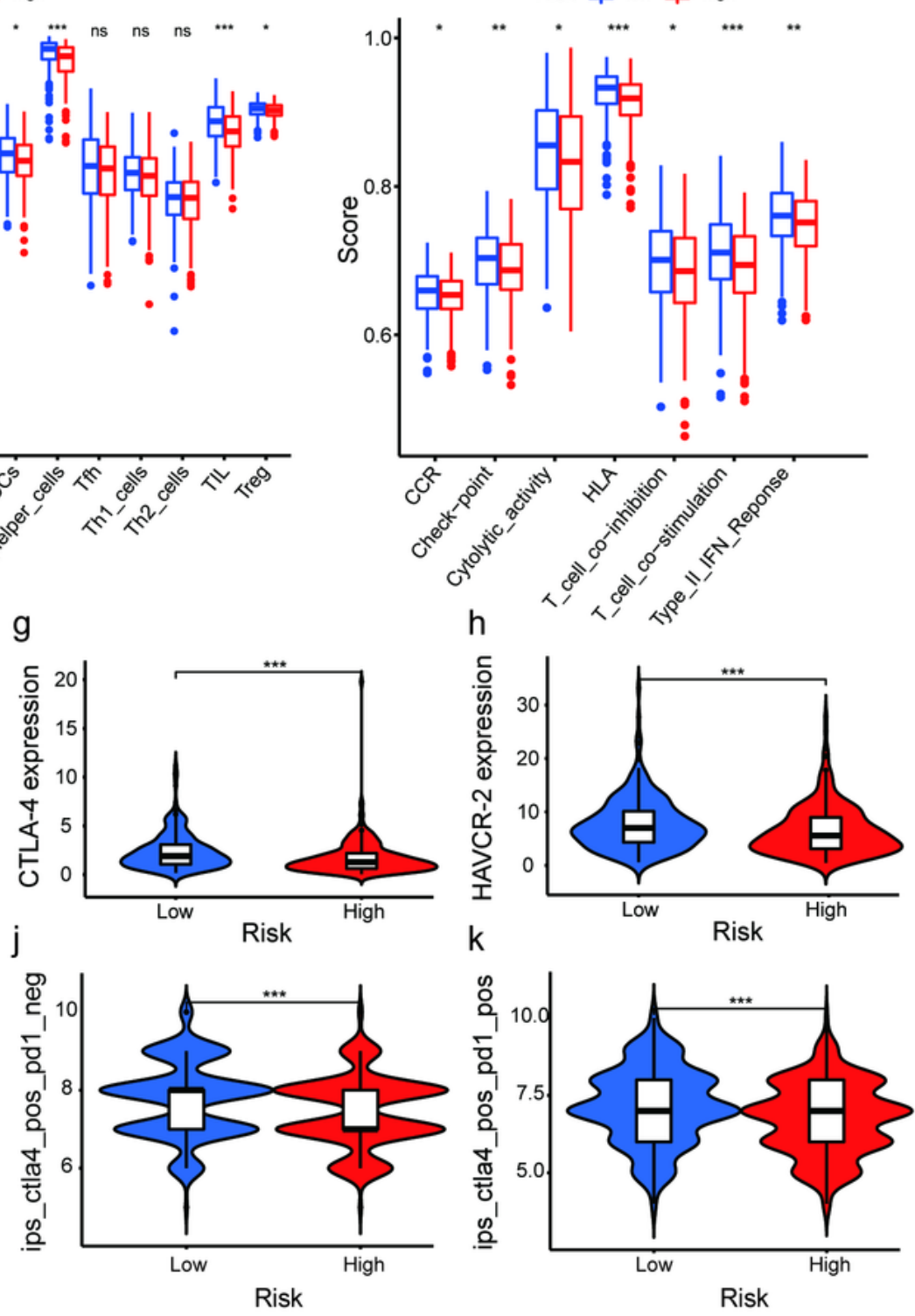

Figure 6

Exploration of the tumor immunity and ICls efficacy of the ARIncRNA signature. (a-c) The column diagrams reveal that low-risk group patients have a higher StromalScore (a), ImmuneScore (b), and ESTIMATEScore (c). ( $d$ and e) The multi-boxplots show that patients in low-risk group have a higher 
abundance of aDCs, B cells, DCs, iDCs, mast cells, neutrophils, pDCs, T helper cells, TIL, and Treg (d). Several immune pathways, including CCR, check-point, cytolytic activity, HLA, T cell co-inhibition, T cell co-stimulation, and type II IFN response were enriched in low-risk patients (e). (f-h) The violin plots indicate that high-risk score correlated significantly negatively with high PD-1 (f), CTLA-4 (g), and HAVCR2 (h) expression, revealing low-risk patients are more applicable for ICls treatment. (i-k) The violin plots validate the results above that regardless of whether it is a PD-1 blocker alone (i), a CTLA-4 blocker alone (j) or a combination of the two $(k)$, the efficacy of patients with low-risk is better than that of high-risk patients. 